\title{
Probando sentidos: discursos sobre drogas dentro de tribus urbanas
}

\author{
Ana María Guerrón ${ }^{1}$
}

RESUMEN

LAS DROGAS SON VISTAS, MÁS ALLÁ DE SUS EFECTOS FISIOLÓGICOS, COMO PRODUCTOS LLENOS DE CONTENIDOS QUE SE HAN IDO CONSTRUYENDO A TRAVÉS DE LA HISTORIA DE CADA PUEBLO Y COLECTIVO SOCIAL. ESTÁN RODEADAS Y HAN CREADO TANTO REALIDADES COMO IMAGINARIOS SOCIALES, DENTRO DE LOS CUALES SE INSERTAN SUS CONSUMIDORES. EN EL CONTEXTO DE DOS TRIBUS URBANAS: PUNKS Y HARDCORES, SE ANALIZAN LOS DISCURSOS DE LAS MISMAS SOBRE EL CONSUMO, CON EL OBJETIVO DE ENTENDER SUS REPERCUSIONES EN LA CONSTRUCCIÓN DE IDENTIDADES INDIVIDUALES Y COLECTIVAS.

Palabras clave: Drogas-tribus urbanas-punKs-hardocores

\begin{abstract}
Amstract
Drugs ARE SEEN BEYOND THEIR PHYSIOLOGICAL EFFECTS, AS CONTENT-FILLED PRODUCTS THAT HAVE BEEN BUILT THROUGH THE HISTORY OF EVERY PEOPLE AND SOCIAL GROUPS. THEY ARE SURROUNDED AND HAVE CREATED BOTH, SOCIAL IMAGINARIES AND REALITIES WITHIN WHICH, CONSUMERS ARE INSERTED. IN THE CONTEXT OF TWO URBAN TRIBES: PUNKS AND HARDCORE, WE ANALYZE THE SPEECHES OF THE SAME CONSUMPTION, IN ORDER TO UNDERSTAND ITS IMPACT ON THE CONSTRUCTION OF INDIVIDUAL AND COLLECTIVE IDENTITIES.
\end{abstract}

KEYWORDS: DRUG-URBAN TRIBES-PUNKS- HARDCORES

1 Correo electrónico: anaguerron@gmail.com Licenciada en Antropología Social, P.U.C.E. 


\section{Introducción}

$\mathrm{E}$ l siguiente artículo es una síntesis del último capítulo de mi tesis de licenciatura, en la que analicé comparativamente dos tribus urbanas quiteñas: los hardcore y los punks, enfocándome en el papel que juegan sus consumos de música, de estilos y de drogas en la construcción de su identidad colectiva, partiendo de la descripción del contexto urbano quiteño, uno cambiante y sectorizado, que cuenta con la presencia de nuevos actores. En este escenario, examinamos a las tribus urbanas desde el concepto de la "neotribalización", que está directamente relacionado con el proceso de construcción de la identidad en la época actual. A partir de esta discusión teórica, nos adentramos en el tema de la música, entendida no sólo como una expresión artística, sino como una práctica que se convierte en marca y referente identitario de las agrupaciones juveniles. De la misma manera tratamos al estilo, que representa una forma de manifestación y de apropiación del cuerpo, así como también una construcción del mismo que parte del consumo. Finalmente, pasamos al análisis de los discursos que giran en torno a las drogas y a la relación que su uso tiene en el proceso de identificación dentro de las tribus urbanas, los imaginarios que giran en torno a éste y sus repercusiones sociales. En el presente artículo, más allá de verlo como un tabú, nos interesa enfocarnos en los aspectos sociales que rodean estos consumos de drogas y sus consecuencias.

\section{Consideraciones generales}

"De la Antigüedad nos llega un concepto (de la droga)-ejemplarmente expuesto por el griego phármakon- que indica remedio y veneno. No una cosa u otra, sino las dos inseparablemente. Cura y amenaza se solicitan recíprocamente en este orden de cosas” (Escohotado, 2004a:20).

La gran variedad de sustancias psicoactivas que se consumen en estos tiempos han dado origen a una serie de imaginarios en torno a quienes las utilizan. De esta manera, el consumo de drogas se ha vuelto un factor considerable a la hora de hablar sobre la construcción de identidades, especialmente entre los y las jóvenes. Si bien hasta principios del siglo XX muchas de ellas eran sustancias consumidas para calmar dolencias físicas (Escohotado, 2004b:20), actualmente su principal uso es el recreativo, muy a pesar de todas las normativas de prohibición impuestas por el Estado.

El consumo de drogas además, ocupa un lugar en el imaginario social que depende del papel que estas sustancias hayan tenido a lo largo de la historia de cada colectivo. En muchas sociedades, por ejemplo, el consumo es parte de ritos de paso o prácticas medicinales que permiten adquirir nuevos saberes místicos. Sin embargo, en nuestro caso de estudio, el contexto se ve atravesado por los cambios que la modernidad ha acarreado y que son fortalecidos por las nuevas tecnologías y los medios de comunicación masivos. Así, nos enfrentamos a una pluralidad de opciones de estilos de vida, ${ }^{2}$ de valores y a un marcado individualismo aislante. Según Ehrenberg (1994), las drogas resultarían instrumentos que crean individuos y expresan también la libertad individual (independencia y autonomía) limitada por cuestiones políticas al ser usadas para cambiar estados de conciencia. El consumo sería una búsqueda de un lugar en la sociedad democrática, sin intermediarios (ni dioses, ni instituciones jurídicas) que consolidan la aclamada "libertad individual". En este mismo contexto, Tenorio (2002) enfoca al consumo de drogas

2 Definidos por Giddens como “un conjunto de prácticas más o menos integrado que un individuo acoge, no sólo porque estas prácticas satisfacen necesidades utilitarias, sino porque dan una forma material a una narrativa particular de la identidad" (1991:84). 
como un medio de construcción de saberes y afectos que brindan a quienes las usan sentidos, que influencian la manera de ver y relacionarse tanto con el mundo como con ellos mismos. Además, se plantea que las drogas son para sus usuarios, un "ícono dispuesto a ensamblar en su ser realidades múltiples, convergentes y contradictorias” (Tenorio, 2002:70), contrastando con el "déficit metafórico" del usuario. Según el autor, las drogas constituyen certezas dentro de un "mundo en conflicto" o de un usuario que vive en la incertidumbre con respecto a sus opciones de vida o una (supuesta o no) marginación social que enfrentan. De esta forma, se irían construyendo "redes" de consumidores en las que se halla un acuerdo y sobre todo, un sentido específico. Sin embargo, cabe mencionar que estos principios se aplicarían sobre todo a personas adictas, más no a usuarios esporádicos como son la mayoría de miembros de las tribus urbanas de este caso de estudio.

Otros estudios (Cerbino, Chiriboga y Tutivén 2001; Guerrero, 1998; Sepúlveda, 1997; Tenorio, 2002), sitúan a las drogas como creadoras o recreadoras de espacios de socialización, con una ritualidad y un contexto específico. En este caso, cada droga se vuelve una herramienta de identificación o una marca identidaria ${ }^{3}$ que ubica a una persona dentro o fuera de un grupo social, debido a que poseen un contenido simbólico, una significación cultural y también un uso dentro de un imaginario (en este caso el juvenil). Es decir, ya se ha creado un estereotipo consolidado tanto de la droga como de quien la consume. ${ }^{4}$

Con estos antecedentes, haremos un recorrido por los discursos que manejan ambos grupos alrededor de algunas drogas, cuya compra y venta es bastante generalizada en el país, pese a que su consumo y tenencia sea ilegal. La facilidad para ser obtenidas ha aumentado considerablemente el número de sus consumidores en los últimos años, así como la cercanía e información sobre las mismas, ${ }^{5}$ dando como resultado una juventud más enterada al respecto (en comparación con la de sus padres) y por tanto, con razones que ya no sólo se basan en un tabú a la hora de elegir o no su consumo.

Finalmente, hay que notar que ninguna de las drogas consumidas por los jóvenes hardcore o punk, se ha librado de los estereotipos creados a través de su historia y por lo tanto, sus consumidores tampoco. Más allá de las consecuencias personales que conlleva su uso, existen algunas drogas sociales, claramente visibles en la sectorización de los discursos juveniles.

\section{Discursos y prácticas en torno al consumo de drogas}

Como se ha mencionado previamente, la significación que tienen las drogas varía de acuerdo al tipo de identidad juvenil y a las representaciones que se han construido a través de la historia. Por ende, tienen una carga cultural que es trasmitida al consumidor. De ahí que los discursos que giran en torno a las drogas constituyan un camino válido para la comprensión de los consumos, los cuales, denotan clasificaciones y órdenes de la realidad así como las valoraciones del consumo y sus efectos. Además, en el discurso se expresan metáforas, que son narrativas relacionadas con saberes. Los discursos que giran en torno a las drogas, expresan rasgos de la identidad, recrean prácticas y dibujan un mapa social que contiene estereotipos, realidades y encadenamientos lógicos de los sentidos con los que están cargadas simbólicamente cada sustancia.

3 De la misma forma que sucede con la música y con el estilo de vestir dentro de las tribus urbanas.

4 Según Bernardo Guerrero (1998), es en el consumo de una droga que los jóvenes que no se sienten identificados con algunas estructuras intermedias de la ciudad (el barrio, por ejemplo), encuentran un sentido y una identidad: "este consumo los ubica dentro de una categoría cultural (...) que vista desde fuera es un estigma, pero que a sus propios ojos, resulta aglutinadora” (Guerrero, 1998:46).

5 En gran parte gracias a medios de comunicación como el Internet, ya que otros como la televisión todavía mantienen un discurso satanizante y generalizador sobre "la droga". 
En nuestro caso de estudio, el discurso acerca de las drogas estaba muy definido por ambas tribus urbanas ${ }^{6}$ mostrando que en su imaginario, cada una de ellas tiene un lugar específico que depende de las realidades que han ido creando. Para los hardcores, a diferencia de los punks, sí hay límites marcados en cuanto al consumo de otras drogas (con excepción de la marihuana). ${ }^{7}$ Por otro lado, ambos grupos piensan que en la actualidad el consumo es tan generalizado que entre ellos ha crecido la tolerancia. Por ejemplo, una entrevistada punk plantea: "Es como que hay tanta propaganda para las drogas (...). En mi época, la propaganda era: 'te drogas y eres un perdedor, puta te cagaste la vida, eres una mierda'...” (Entrevista a A.G.). Notamos que los medios de comunicación también influyen en la creación de estos discursos, reforzando estereotipos. Incluso desde hace más de dos décadas, existen películas ${ }^{8}$ sumamente populares, en las que el consumidor es mostrado como una persona que ha reflexionado sobre su uso y logra presentar ventajas o argumentos adecuados sobre éste; o se muestran personajes totalmente estereotipados, hasta cierto punto burlesque, que a pesar de su consumo mantienen un ritmo de vida normal y no terminan en un centro de rehabilitación.

En todo caso, el tabú se ha ido desvalorizando, pasando a ser más cercano a una patología ${ }^{9}$ que a problemas delincuenciales, especialmente entre los y las jóvenes. A continuación realizaremos un rápido recorrido de los discursos de ambos grupos y su visión de cada droga o el lugar que ésta ocupa en su imaginario.

\section{Cannabis Sativa}

La marihuana ${ }^{10}$ goza de un lugar priviligiado entre los hardcore y los punks, principalmente por sus efectos pero también porque es considerada inócua. Según los jóvenes, la sociedad quiteña todavía tiende a relacionar al consumo de marihuana con la delincuencia, la violencia ${ }^{11}$ o problemas en el hogar. Aún así, los imaginarios hardcores son distintos y mucho más cercanos al discurso y mensaje que promueve el movimiento, por lo que esta es la droga con la que más se identifican. ${ }^{12}$ Aún así, es interesante notar que les anexa con una identidad "rebelde" o provocadora, que reproduce actos contrarios a la sociedad de la que forman parte. Además, esta particularidad se relaciona con la identidad hardcore, que tampoco tenía connotaciones positivas en el imaginario social de la ciudad y que aún así constituye para ellos una fuente de sentido. En este punto cabe mencionar el conflicto interior que se da al oponerse con su consumo a la sociedad adulta y especialmente a sus padres, algo que es mencionado en su discurso y justificado al mencionar que las consecuencias del consumo para ellos han sido mucho más positivas que negativas. En este sentido, el consumo se da como una reivindicación de un sujeto dueño de sí mismo:

"Se ejerce poder sobre su propio cuerpo, que se desliga del control de las generaciones adultas (...) el consumo deviene una táctica para dominar el propio cuerpo, así como, construir nuevos saberes que se convierten en bienes preciados y sumamente importantes al sentirlos como íntimos y originales” (Guerrón, 2009:114).

6 Seguramente debido a que todos los entrevistados tenían o han tenido algún contacto con el consumo de drogas. No necesariamente las han probado, pero a ninguno le eran totalmente desconocidas o ajenas.

7 Existe también una división dentro de los hardcore, llamada Straight Edge, sus miembros no usan ni toleran el uso de ninguna droga.

8 Como “Easy Rider” que fue seguramente una de las primeras. También “Trainspotting” se convirtió en una película de culto para los jóvenes que vivieron en los años 90s.

9 Se ha desarrollado un discurso que parte desde el ámbito de la salud y la medicina y ya no desde lo jurídico de lo legal.

10 Marihuana, weed, "hierba”, “grifa”, “ganlla”.

11 Irónicamente, ya que sus efectos son más bien tranquilizantes.

12 Si bien su consumo no es una condición para la entrada al grupo ni tampoco todos aquellos que participen en una banda la consumen. 
Creándose con este consumo un "espacio autogobernable”, muy parecido al que han construido con sus colegas de banda o sus amigos en conciertos. Tanto en conciertos de hardcore como de punk el consumo de marihuana está presente y usualmente es compartido. Si bien éste no es promocionado, ${ }^{13}$ tampoco se lo oculta. De todas maneras, seguramente debido a los efectos relajantes que se le atribuyen a la marihuana, su consumo se da sobre todo después del concierto (que es un momento de intensidad, muy energizante). Además "esta droga, a diferencia de otras, tiene un uso doble: por un lado, aglutina al grupo en el momento del consumo, y por otro lado, les permite adentrarse en sí mismos” (Íbid, 2009:112).

Por otro lado, entre los punks el consumo se ha "desvalorizado", creemos que por un lado debido a lo frecuente ${ }^{14}$ que es entre todo tipo de jóvenes, y también a sus efectos "suaves” (en comparación a otras drogas), que lo ha convertido en un consumo menos "marginal”. ${ }^{15}$ Por tanto, en su imaginario este consumo es algo incluso normal: "Hay todo tipo de gente que consume marihuana. No hay estereotipos para la marihuana, no hay clases sociales, no hay nada. Toda la gente consume” (Entrevista a R.G.).

En general el primer consumo se da con otros jóvenes del grupo que tienen mayor tiempo experimentandolo y que en muchos casos, luego se convierten en sus proovedores. ${ }^{16}$ Es en estos primeros encuentros con la marihuana cuando los jóvenes aprenden todas las técnicas que la rodean, por ejemplo armar un "porro"17, "descogollar"18, etc.:

"El o la neófita es aceptado/a e iniciado/a por los y las consumidores/as experimentados/as, este aspecto participa incluso de la imagen del producto y de sus usuarios/as: comunión, reparto, igualdad, libertad, sin juicio de valor” (Le Garrec, 2002:133).

Es por esto que es un consumo que consta de toda una ritualidad. H. Becker señala que hay tres etapas: "el aprendizaje de la técnica, de la percepción de los efectos, del gusto por los efectos” (Íbid, 2002:136). Estableciéndose en este punto diferencias notables entre cada usuario, que confirman siempre su autenticidad, la misma que determinará incluso el grado de amistad que se mantenga con otro miembro del grupo. Por ejemplo, para un fumador experimentado: "Los que fuman marihuana de verdad, sólo fuman marihuana. (...) Yo no conozco a nadie que fume enserio marihuana, no de la forma de verlo así: "Fumemos porque sí, y ya" (Entrevista a T.P.). Parte de la ritualidad también es llevar consigo el "equipo" (marihuana, una pipa, fosforera, etc.), conocer la calidad del producto y la resistencia que se tenga a los efectos de la marihuana, también pueden marcar una diferenciación con los principantes. De todas formas, es en la cotidianidad que se van reconociendo, y marcando los elementos que determinan si el consumidor es parte del grupo. Algo muy similiar a lo que sucede cuando comienza su aprendizaje musical, ya sea el de tocar un instrumento o el de tener un conocimiento amplio sobre la música hardcore o punk, algo imprescindible para formar parte del grupo y sobre todo, para mostrarse como auténtico hardcore o punk. Se trata entonces de franquear diferencias y

13 Como lo es en la música reggae o hip-hop.

14 Incluso por los “aniñados”, sus opuestos. La clasificación de “aniñados” se da en Quito, desde el discurso de los hardcore y punks, para los jóvenes que pertenecen o aparentan pertenecer a una clase socioeconómica alta, que visten con la moda de la cultura dominante, vendida en grandes tiendas, frecuentan lugares lúdicos en donde el precio de entrada es más alto que el promedio, y escuchan cualquier otro tipo de música que no sea hardcore o punk. En síntesis, se puede decir que un “aniñado” es alguien que no presenta ninguna actitud crítica con la moda y los mensajes vendidos por los medios masivos.

15 Si tomamos en cuenta que la identidad punk se construye desde la "automarginalidad" que le da un mayor "estatus" a quien la lleve al extremo.

16 En estos casos no suele darse una relación de compra-venta, más bien la marihuana suele ser obsequiada por quienes siembran sus propias plantas, o por quienes la compran a dealers, es decir vendedores "al por mayor" de marihuana y otras drogas, los mismos que no pertenecen al medio hardcore.

17 Un cigarrillo armado con papel de arroz que contiene marihuana.

18 Moler el cogollo seco de la planta de marihuana. 
afianzar relaciones que al principio son desiguales, complementando la "comunidad emocional" que se construye entre los hardcores y punks. De todas formas, es un consumo que marca el cuerpo con el aprendizaje de nuevos saberes (de léxico, de técnicas, etc.) que los caracteriza dentro de su grupo hardcore o punk.

\section{Alcohol}

Debido a su generalización y legalidad, la constancia con la que se ha dado su consumo y el aspecto lúdico con el que es identificado, no es usualmente visto como una droga entre los jóvenes. Incluso se ha ido constituyendo el personaje del "borracho" reconocido en múltiples sociedades, por lo que el consumo de bebidas alcohólicas no sale de la "normalidad": "El individuo antes de encontrarse con un vaso de alcohol en la mano vive en un ambiente en donde la alcoholización es frecuente. El alcohol tiene un lugar importante en el espacio público" (Íbid, 2002:121). En el país, el consumo es extendido y los y las jóvenes son asiduos bebedores. ${ }^{19}$

Sin embargo, hay una gran diferencia del consumo entre hardcores, en cuyos conciertos no es tan frecuente, y punks, entre quienes es casi necesario. Como lo explica un entrevistado hardcore, estar ebrio es volverse "un libro abierto", es perder el control, estar a disposición del resto. El consumo expone al cuerpo, “el alcohol nos lleva hacia el afuera, los alucinógenos nos traen hacia nosotros mismos” (O. Paz citado en Le Garrec, 2002:127). Esto conlleva a olvidarse del ser, a anularlo, algo totalmente contrario al mensaje del discurso hardcore que contiene un mensaje de lucha y firmeza. Por otro lado, se relaciona más con el mensaje del punk, del No future, de la desesperanza y la valoración sólo del presente y todas las posibles formas para disfrutarlo. Para los hardcore, el que no haya un consumo exagerado de alcohol en sus conciertos es algo que les produce orgullo, ya que, a diferencia de otras tribus urbanas y géneros musicales, son conciertos en los que no hay violencia. En cambio entre los punks el consumo se da en todo momento y lugar en los conciertos: "En los conciertos alcohol es lo que sobra y como es legal es una cultura alcohólica la que vivimos, se hace normal salir todos los días a tomarte una biela, a chupar con tus panas" (Entrevista a R.G.).

El alcohol, entre quienes van a conciertos ya sea a presentarse o como asistentes es una presencia constante, que en su discurso es expresada como un "mal necesario", ya que a pesar de estar conscientes de sus consecuencias y peligros, lo mantienen. Escohotado (2004b) observa que si bien existe "un 'olvido de si', también se marca una "angustiada huida del aburrimiento" que caracteriza al espectador de teatros, la "realidad ebria” (también "realidad dionisíaca”) no admite cosa distinta de la propia vivencia, y conduce a actos de celebración” (Íbid:108). Es un consumo que les permite "vivir el presente". Aún así, según el discurso de los punks, existen aquellos que beben al punto de perder la consciencia y aquellos que no llegan a ese extremo. Ambos tipos se encuentran tanto en conciertos, espacio público en donde se reúna el grupo (incluso en ensayos) o cualquier momento festivo. Muy a diferencia de los hardcore, quienes lo hacen casi exclusivamente después de los conciertos.

19 Según datos del CONSEP: "La prevalencia de vida del consumo de alcohol en el ámbito nacional, en el 2005, es de 61.6\% (...) La edad promedio del primer consumo de alcohol es de 13.7 años” (OED y CICAD-OEA 2005:29-30). 


\section{Pasta base de cocaína}

Esta droga ${ }^{20}$, que es de muy baja calidad y valor, ha sido considerada también una de las más dañinas para el organismo y es altamente adictiva. En el imaginario social de Quito, es asociada a las clases bajas y a la delincuencia; para conseguirla también es necesaria una gestión peligrosa. Es una de las drogas "fuertes” a la que más cercanía han tenido los hardcore, aún si la consideran basura. Sin embargo, en su discurso la mencionan cuando quieren hacer una oposición con la marihuana: la base tiene connotaciones sumamente negativas, mientras que la otra es inofensiva. Entre sus principales efectos, está el de la pérdida del control de sí mismo, que en conjunto con su alta adictividad, hace que ésta se convierta en el centro de su vida: "es levantarte a fumar basuco y esperar el día siguiente para pensar cómo vuelves a fumar (...) O sea esa sería como la real adicción, el real drogadicto" (Entrevista a T.P.). Notamos que a diferencia de la marihuana que para ellos no constituye un problema verdadero, ${ }^{21}$ la pasta base de cocaína implica “envolverse en 'el mundo de las drogas', es decir en una adicción de la que seguramente no ven ninguna salida, una adicción que es real, es objetiva” (Guerrón, 2009:121). Y no sólo tiene una relación con problemas de salud, sino también con la delicuencia y la vida callejera, algo ajeno a su medio y con lo cual no se identifican: “...la gente que pertenece ya a las clases más populares, por lo general son basuqueros antes que marihuaneros. Por el mismo hecho de las actividades que ellos tienen, muchos roban, o sea muchos están en la calle, la calle mismo les pudre" (Entrevista al grupo CDL).

Para los punks también es la droga antítesis de la marihuana pero por la dimensión social del usuario: "Es que el basuco es muy individualista, no es como la hierba que tú compartes, el basuco es sólo para ti y estás escondiendo, porque nada es suficiente, y tienes que estar encerrado solo" (Entrevista a A.G.). Debido a sus efectos poco "sociables", no es muy frecuente en los conciertos. Los punks mayores comentan que debido a todas las implicaciones que tiene, sólo podría consumirlo alguien que ya lo ha dejado todo y lleva una vida "callejera” (como algunos punks mayores lo hicieron). No es una actividad lúdica entonces, como otros consumos: "o sea para mí y para la gente que me rodeaba, el basuco era como demasiado íntimo (...) Si tú fumas en un concierto, eres un chamo (...) porque un crónico no hace esas cosas" (Entrevista a P.A.).

Así como lo expresa el argot que la denomina “pistola”, los relatos de los y las jóvenes también expresan lo peligroso de esta droga, porque es una que puede matar y que constantemente amenaza con hacerlo. Lleva a quien la consume a perder todo interés por otras actividades (incluyendo la pasión de estos/as jóvenes, la música): “Te vuelves un ente”, relataban, no sólo mata al organismo del quien la consume, sino también sus relaciones intragrupales.

\section{Pegamento}

Los inhalantes 22 producen efectos distintos a los de las drogas ya mencionadas. Fueron mencionados en sus discursos, especialmente aquellos de punks. Los hardcores por otro lado, no mostraron mucho conocimiento al respecto, seguramente ya que es un consumo también muy relacionado con la vida callejera: "La soluka se lo reconoce porque es de la gente pobre, le hacen a la soluka por que no tienen plata por que no pueden comer, y se pegan a la funda" 23 (Entrevista a J.C.).

20 A la base de cocaína se la conoce como: “basuco”, "basú”, “base” o "pistola”. Esta droga se consume casi exclusivamente en Sudamérica.

21 Seguramente porque no produce una adicción física ni causa sobredosis (Escohotado, 2004b).

22 Cemento de contacto: "gale”, solución, "soluka” o "el tarro".

23 En referencia a la técnica usada para consumir pegamento: ponerlo en una funda para luego inhalarlo. 
Aunque se puede decir que el consumo entre los punks más jóvenes ha disminuido, todavía se hace presente entre los más radicales o los también llamados “podridos”. Aún así, es un consumo tolerado en el grupo principalmente debido a que sus efectos no tienen tantas repercusiones en la vida social de la tribu urbana: "Esos (los “soluqueros”) sí son tranquilos, no 'pintan un culo"24 (haciendo contraste con los "basuqueros")" (Entrevista a R.G.). Los punks mayores que fueron usuarios le tienen un "afecto" especial, ya que los introdujo en el mundo underground, de nuevos contenidos lleno de alucinaciones y sensaciones. Sin embargo, fue un consumo momentáneo que no extendieron (a diferencia de otras drogas) debido a que eran conscientes de los efectos sumamente perjudiciales para su salud.

\section{Cocaína}

Esta droga de valor elevado, su uso no es tan generalizado en toda clase social y etaria. Entre los hardcores, su uso no es frecuente, y en su discurso la sitúan como una droga de alto riesgo debido a la dependencia física que causa, por lo que clasifican a sus usuarios como gente con falta de fuerza de voluntad al convertirse en esclavos de la misma. También es vista como la droga de los "aniñados” y de gente adinerada. Uno de ellos comenta: “...para mí la cocaína si es como la droga del trabajo, la droga del capitalista por excelencia y, por una cuestión más ideológica, prefiero alejarme de eso. Porque obviamente, ¿quién consume cocaína? O sea, los gerentes, los empresarios, los manes de guita”25 (Entrevista a C.D.L).

Según los punks, en cambio, es una droga peligrosa por lo agresiva que se vuelve la gente que la consume. Si bien antes no era tan frecuente en su grupo, actualmente es mucho más accesible y usada por los nuevos punks: “a mi también me gusta, me parece como que te sientes bacán, experimentas nuevas sensaciones, más bacanes que con la marihuana” (Entrevista a R.G.). Aún así, el alto precio de cada gramo de cocaína continúa impidiendo a la mayoría de los punks consumirla e incluso identificarse con ella.

\section{Conclusiones: las repercusiones}

Si bien los miembros de ambas tribus urbanas no son considerados como adictos ${ }^{26}$, el consumo tiene repercusiones que se notan en su cotidianidad. Sobre todo en la dimensión individual: se establece una relación "personal” entre el usuario y el producto, vinculada a los efectos de cada droga: "Sus usuarios tienen en cuenta el placer que les pueda producir y no los daños que a corto o largo plazo causa, de los que además, están conscientes la mayoría de las veces”27 (Guerrón, 2009:129). Por otro lado, está la dimensión social: se crean relaciones y redes con el resto de consumidores, que parten del mismo consumo y se construyen en torno a él, adscribiéndose de esta forma a un grupo, imaginarios y discursos determinados.

Cabe mencionar, que lo que buscan en cada sustancia es el placer por lo prohibido, que no se da a manera de simple capricho sino que se trata de una relación personalizada, según Tenorio: "producida desde la metaforización de la cosa que deja de ser tal para convertirse en algo mucho más significativo que la varita mágica de los cuentos de hadas” (Tenorio, 2002:70). Tanto para los punks como para los hardcores, esta es una relación que debe ser especial y jamás irreflexiva;

24 No importan.

25 Dinero.

26 Que están totalmente dominados por el consumo.

27 Para ambos grupos el consumo de drogas es igual o tan nocivo como otras prácticas: para los punks es peor ser adicto a los videojuegos, el tabaquismo, la televisión, el consumismo, etc., para los hardcores es peor beber alcohol o fumar tabacos que consumir marihuana. 
lo que se expresa, por ejemplo, en el uso de marihuana y la experiencia introspectiva que les proporciona. Además, cada consumo ha adquirido su momento, lugar, compañía, cantidad, etc. Tenorio agrega: "Parecería que en el sujeto usador se da un déficit metafórico previo que lo conduce a suplirlo mediante la droga cuyo cuerpo contaría, por el contrario, con un exceso de metaforización en la medida en que en ella se busca y se encuentra todo" (Tenorio, 2002:70).

Si bien el consumo en nuestro caso de estudio no se da como una respuesta mecánica a todo, sí funciona como un aditivo. Por ejemplo, en el caso de los hardcore, la marihuana es utilizada como un complemento en la creación musical; para los punks en cambio ésta es un mediador con la música y el resto del grupo. De la misma forma sucede con el resto de drogas consumidas, cada una tiene un momento ya que según este se encuentran las sensaciones buscadas. Más allá de una simple funcionalidad, pensamos que en cada consumo se conocen nuevas metáforas con las cuales se sienten identificados, adscritos y socializados dentro de una tribu urbana. El consumo de marihuana especialmente, que es generalizado en ambos grupos debe ser interpretado como una búsqueda de "otras formas de reflexión y conocimiento de su cuerpo, y por ende, de su proyecto identitario, que conlleva y facilita a su vez, otras formas de socialización” (Guerrón, 2009:132). Esta relación de "compañía” que se crea con una droga, especialmente con la marihuana, es leída por sus usuarios como una relación con un ser que interviene en su cotidianidad, debido a las sensaciones e ideas que les ha causado, que se han quedado en su memoria y que reconstruyen su subjetividad. En el caso específico del consumo de pegamento, por parte de los punks, notamos que esta relación es incluso más cercana e íntima. De todas formas, son experiencias que van creando una coherencia entre lo que "es" y lo que "debería ser", es decir, constituyen sentido en un momento dado incluso si no son parte de su mundo "objetivo".

Por otro lado, muchas veces esta relación con las drogas se vuelve inconveniente. Según los hardcores, los efectos de la marihuana pueden manifestarse o extenderse en pereza para cualquier otra actividad, algo que es una incoherencia con la ética que manejan. Para los punks también es complicado, especialmente cuando trabajan o estudian, ya que deben responder a estas obligaciones, mientras comparten prácticas totalmente opuestas con su tribu urbana. Además están los casos de algunos jóvenes que no controlaron su consumo, prefiriéndolo a sus relaciones grupales y a su interés musical e incluso tuvieron períodos en centros de rehabilitación. ${ }^{28}$

De todas formas, el consumo de drogas es un rompimiento con muchas estructuras sociales, entre ellas también la familia. Una vez que empieza el consumo, se produce un desencuentro: por un lado, los y las usuarias han tomado un camino en el que hallan nuevas imágenes y sensaciones (no sólo con las drogas sino también con la música) a partir de las cuales comienzan a construirse y por otro lado, deben enfrentar muchas veces el rompimiento de los lazos familiares, especialmente con sus padres quienes validan un discurso oficial ${ }^{29}$ que rechaza ambas prácticas.

En cuanto a la dimensión social, recordemos que las drogas más consumidas son tanto la marihuana como el alcohol, que en los conciertos nunca se consumen sin compartir. En el caso de otros momentos, como los ensayos, también se da un consumo colectivo, ya que según dicen, es en estos espacios colectivos, en los que más disfrutan de este porque se comparte también el mismo lenguaje y los mismos saberes. Como comentan, en especial los hardcores, cuando se comparte marihuana con otros, ellos se reconocen e identifican. Su relación se afianza más aún cuando comparten el mismo ámbito musical, razón por la cual son importantes los momentos que suceden afuera de los conciertos, en donde los jóvenes fuman marihuana. En cuanto al alcohol, a veces crea conflictos internos dentro del grupo, de punks especialmente, ya que puede ocasionar escenas de violencia, pero en general, también se trata de un consumo compartido, que establece nuevas relaciones sociales.

28 Lo cual tiene connotaciones negativas en su discurso, ya que este es un proceso que determina que la persona cortó también relaciones con el grupo y tal vez más importante aún, con su interés musical.

29 Tanto político, médico, jurídico y mediático. 
En medio de discursos oficiales contradictorios sobre las drogas y los de cada usuario de ellas, los y las jóvenes se encuentran en una paradoja en el consumo: por un lado buscan interiorizar la experiencia y por otro, se reúnen en grupo para hacerlo, mientras se excluyen de una sociedad que marginaliza estas prácticas. Esto nos recuerda a la característica de las tribus urbanas (Maffesoli, 2000) de “individualización colectiva”. El etiquetaje social parte de quienes buscan ser individuales, más en este camino terminan homogeneizándose, y a la vez compartiendo saberes y realidades que poco a poco van estableciendo y construyendo relaciones afianzadas, en medio de un contexto que a sus ojos está conformado por otros grupos e instituciones con las cuales nunca se identificarán. Esta es también una manera desafiante de enfrentar su medio, ya que parte de lo prohibido, pero a su vez replantea al consumidor que muchas veces llega a posicionarse como creador de nuevos saberes y, lastimosamente en los peores casos, puede llegar a negarle su existencia social e individual. 


\section{Bibliografía}

Cerbino, Mauro, Cinthia Chiriboga, y Carlos Tutivén. 2001. Culturas juveniles. Cuerpo, música, sociabilidad y género. Primera edición. Quito: Convenio Andrés Bello y Ediciones Abya-Yala.

Ehrenberg, Alain. 1994. Individuos bajo influencia. Drogas, alcoholes, medicamentos psicotrópicos. Buenos Aires: Editorial Nueva Visión.

Escohotado, Antonio. 2004a. Historia de las drogas. España: Alianza Editorial.

Escohotado, Antonio. 2004b. Historia de las drogas. España: Alianza Editorial.

Giddens, Anthony. 1991. Modernity and self-identity. Self and society in the late modern age. California: Standford University Press.

Guerrero, Bernardo. 1998. Hasta Que El Cuerpo Aguante. La dinámica socio-cultural del consumo de drogas. Chile: Editorial El Jota Errante.

Guerrón, Ana María. 2009. Construcción de Identidades dentro de tribus urbanas. Consumos de músicas, estilos y drogas. Tesis de disertación de grado, Escuela de Antropología, Quito: Pontificia Universidad Católica del Ecuador.

Le Garrec, Sophie. 2002. Ces ados qui "en prennent”. Sociologie des consommations toxiques adolescentes. Toulouse: Presses Universitaires du Mirail.

Maffesoli, Michel. 2000. Le temps des tribus. Le déclin de l'individualisme dans les sociétés postmodernes. Tercera edición. Paris: La Table Ronde.

OED, Observatorio Ecuatoriano de Drogas, and Comisión Interamericana Contra el Abuso de Drogas CICADOEA. 2005. "Segunda Encuesta Nacional a Estudiantes de Educación Media sobre Consumo de Drogas.”

Sepúlveda, Mauricio. 1997. "El silencio de los angustiados: contextos discursivos en el consumo de la pasta base de cocaína.” in La Grieta De Las Drogas. Desintegración social y políticas públicas en América Latina, edited by Martin Hopenhayn,. Chile: Naciones Unidas.

Tenorio, Rodrigo. 2002. Drogas. Usos, lenguajes y metáforas. Quito: Abya-Yala. 